\title{
Prognostic value of excision repair cross-complementation group 1 expression in gastric cancer: A meta-analysis
}

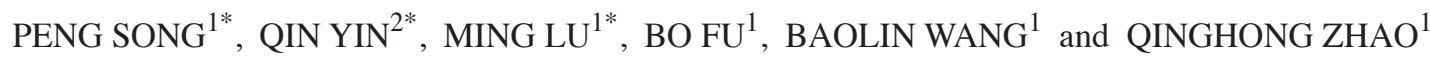 \\ ${ }^{1}$ Department of General Surgery and ${ }^{2}$ Medical Center of Pediatrics, \\ The Second Affiliated Hospital of Nanjing Medical University, Nanjing, Jiangsu 210011, P.R. China
}

Received May 14, 2014; Accepted January 14, 2015

DOI: $10.3892 /$ etm.2015.2284

\begin{abstract}
The prognostic impact of excision repair cross-complementation group 1 (ERCC1) expression in gastric cancer (GC) has been investigated for decades, but has yielded controversial results. The aim of the present study was to provide a precise evaluation of whether the expression levels of ERCC1 are associated with overall survival (OS) in patients with GC. A systematic search of Medline and Embase was conducted. Original studies concerning OS and ERCC1 expression were included for critical appraisal. A total of 15 studies comprising 1,425 patients with GC were identified. The results revealed that high/positive ERCC1 expression was an indicator of poor survival in patients with GC [hazard ratio (HR) 1.48; $95 \%$ confidence interval $(\mathrm{CI}) 1.02-2.10 ; \mathrm{P}=0.036 ; \mathrm{I}^{2}=83.8 \%$; random-effects model] compared with low/negative ERCC1 expression. Subgroup analysis indicated that high/positive ERCC1 expression had a significant unfavorable impact on OS in the group of patients evaluated by reverse transcription polymerase chain reaction (RT-PCR; HR 2.57; 95\% CI 1.49-4.45). Furthermore, high/positive ERCC1 expression was found to be associated with poor survival in patients receiving platinum-based chemotherapy in the RT-PCR group (HR 2.13; 95\% CI 1.06-4.27). These data suggest that ERCC1 may be a useful prognostic factor for GC. In addition, low mRNA levels of ERCC1 appear to be associated with a significant favorable OS benefit from platinum-based chemotherapy.
\end{abstract}

Correspondence to: Dr Qinghong Zhao, Department of General Surgery, The Second Affiliated Hospital of Nanjing Medical University, 121 Jiang Jia Yuan Road, Nanjing, Jiangsu 210011, P.R. China

E-mail: njzhqh@sina.com

${ }^{*}$ Contributed equally

Key words: excision repair cross-complementation group 1, gastric cancer, meta-analysis

\section{Introduction}

Despite the fact that the incidence of gastric cancer (GC) has decreased substantially over the past few decades, it remains the fourth most common cancer and the second leading cause of cancer-related mortality worldwide (1). Generally, surgical resection with adjuvant chemotherapy is used to treat GC (2); however, the overall survival (OS) remains poor and no standard treatment has been determined. Discovering new molecular biological prognostic factors could provide a more accurate prediction of clinical outcome and help in the management of patients with GC.

Excision repair cross-complementing group 1 (ERCC1) protein, serving as a rate-limiting enzyme, is a key component in the nucleotide excision repair (NER) pathway (3). The NER pathway functions to remove bulky adducts that are introduced by platinum-containing drugs, such as cisplatin and oxaliplatin $(4,5)$. Platinum-based chemotherapy for GC is one of the most widely used types of anticancer treatment $(6,7)$. Previously, numerous studies have investigated the association between ERCC1 expression and survival in GC (8-10). In brief, they indicate that ERCC1 is not only a prognostic marker for survival but also a predictor of the response to platinum compounds; however the previous studies yielded inconsistent results and no robust evidence. Considering the potential value of ERCC1, a meta-analysis has been conducted to provide evidence-based results on the prognostic and predictive utility of ERCC1 in GC.

\section{Materials and methods}

Search strategy and inclusion criteria. A comprehensive search of Medline and Embase databases was conducted for all relevant literature published in the English language up to April 1, 2014. The medical subject headings for the search were 'ERCC1' and 'gastric cancer'. In order to find additional studies, the references cited in the papers found in the database search were also manually searched. The eligible studies included in this meta-analysis met the following criteria: i) Patients with GC; ii) evaluation of ERCC1 expression and OS; and iii) presented the data for OS or data that allowed the OS to be calculated. When the patient population was duplicated, only the most recent or most complete study was included. 
Data extraction. Two authors independently reviewed all the potentially relevant studies and extracted the data required using standard forms. The following information was collected from the eligible studies: Surname of first author; year of publication; country; sample size; ERCC1 expression assessment method; cutoff values for high/positive vs. low/negative ERCC1 expression; stage; neoadjuvant or adjuvant chemotherapy; the hazard ratios (HRs) and their 95\% confidence intervals (CIs). If HR was not directly reported, the HR estimate and its variance were reconstructed based on published methodology (11). Disagreements were resolved through discussion among the authors.

Quality assessment. The study quality was evaluated by two investigators using the scale reported previously (Table I) $(12,13)$. Briefly, this scale contained seven elements: i) The inclusion and exclusion criteria for patients; ii) the study design; iii) characteristics of the patients; iv) ERCC1 expression ascertainment method; v) the study endpoint; vi) follow-up time; and vii) time lost to follow-up. A high quality element was awarded one point and a maximum of two points was awarded for the method used to measure ERCC1 expression; hence, the maximum score was eight points. Each score provided by a different reader was compared and a consensus was achieved.

Statistical analysis. The individual HRs corresponding to their 95\% CIs were pooled into a summary HR to evaluate the association between ERCC1 level and OS. The significance of the summary HR was measured by aZ-test; $\mathrm{P} \leq 0.05$ was considered to indicate statistical significance. Fixed-effects models were used with the assumption of homogeneity of studies in the first stage. The assumption was examined by assessing the heterogeneity across studies using $\chi^{2}$ test and $\mathrm{I}^{2}$. If the heterogeneity was significant between studies $\left(\mathrm{P}_{\text {heterogeneity }}<0.1\right.$ and $\left.\mathrm{I}^{2}>50 \%\right)$, a random-effects model was performed in the second stage. Additionally, random-effects models were applied in cases where there was qualitative evidence of methodological heterogeneity within studies (e.g., different methods of measuring ERCC1 expression). To explore the possible heterogeneity among different studies, the following key characteristics were examined in a meta-regression model: Study location; ERCC1 expression ascertainment method; sample size; HR estimation method; and quality score. Sensitivity analysis was carried out by sequential omission of each study. Publication bias was evaluated with funnel plots, Begg's test and Egger's test (14). The analyses were performed with STATA software (version 12.0; Stata, College Station, TX, USA).

\section{Results}

Search results, study characteristics and quality assessment. According to the search strategy, a total of 182 articles that mentioned ERCC1 expression and GC were identified. Following the removal of duplicates, 110 abstracts were screened based on the inclusion criteria. Among them, 32 articles remained for detailed evaluation. Seventeen of those 32 articles were subsequently excluded for the following reasons: Review or editorial $(n=5)$; without available data $(n=11)$; or on the same population $(n=1)$. Finally, 15 studies were selected for this meta-analysis (8-10,15-26) (Fig. 1).
The main characteristics of the 15 studies are summarized in Table II. The sample size ranged from 41 to 322. Eleven studies were based in Asia, three in Europe and one in North America. The expression of ERCC1 was evaluated by immunohistochemistry (IHC) in ten studies, and reverse transcription polymerase chain reaction (RT-PCR) in five studies. Different cutoff values of ERCC1 expression evaluation were used. IHC was mainly divided by staining intensity and the percentage of cells stained, whereas ERCC1 mRNA levels were categorized according to median values and maximal $\chi^{2}$ method. In addition, the patients were receiving chemotherapy; most of them were using platinum-based regimens. The quality scores of included studies are summarized in Table II and ranged from 5 to 8 .

Quantitative synthesis. As shown in Table II, 10 of the 15 studies reported that high/positive ERCC1 expression in patients with GC was associated with poor survival, three studies indicated no association between ERCC1 expression and survival, and two studies exhibited an inverse association. Overall, the pooled HR for the 15 studies was 1.48 (95\% CI 1.02-2.10; $\mathrm{P}=0.036$; random-effects model) with significant heterogeneity $\left(\mathrm{P}_{\text {heterogeneity }}<0.001, \mathrm{I}^{2}=83.8 \%\right)$, suggesting that high/positive ERCC1 expression was an indicator of poor survival in patients with GC.

When stratifying by study location, no association between ERCC1 expression and OS was observed in the Asian region (HR 1.54; 95\% CI 0.99-2.38) or the non-Asian region (HR 1.31; 95\% CI 0.63-2.75; Table III). Focusing the analysis on ERCC1 expression ascertainment methods, the pooled HR was 1.09 (95\% CI $0.69-1.72)$ with an $\mathrm{I}^{2}$ of $79.8 \%$ for the ICH group, and 2.57 (95\% CI 1.49-4.45) with an $\mathrm{I}^{2}$ of $82.4 \%$ for the RT-PCR group, respectively (Fig. 2). The significant correlation was also present in the subgroup analysis by sample size $(<100)$, HR estimated (directly obtained) and quality score (7-8) (Table III).

Of the 15 studies, there were 14 reports regarding the OS of patients receiving platinum-based chemotherapy. Meta-analysis of these studies also provided evidence of a trend toward poor survival with high ERCC1 expression (HR 1.33; 95\% CI 0.88-2.00; $\mathrm{I}^{2}=83.6 \%$; random-effects model), although this was not considered statistically significant. In subgroup analysis by ERCC1 expression measurement method, a significant association was observed in the RT-PCR group (HR 2.13; $95 \%$ CI 1.06-4.27) with marked heterogeneity $\left(\mathrm{I}^{2}=84.3 \%\right.$, Fig. 3$)$.

Meta-regression. In univariate meta-regression analysis, only the method used to measure ERCC1 expression $(\mathrm{P}=0.044)$ was found to be a significant source of heterogeneity (Table III); however, the estimated between-study variance $\left(\tau^{2}\right)$ was reduced from 0.410 to 0.403 , which could only explain $1.7 \%$ of the $\tau^{2}$.

Sensitivity analysis and cumulative analysis. Sensitivity analysis was performed to assess the influence of individual studies on the pooled HR. Similar HRs and 95\% CIs were generated by omitting any single study using a random-effects model, and indicated that the results were relatively stable (Fig. 4). A cumulative meta-analysis of the 15 studies was conducted according to the publication date. As 
Table I. Criteria for quality assessment by De Graeff (12).

Score

Criteria

Sub-criteria

Criteria

1. Is the population under study defined with in and exclusion criteria?

1

2. Were patient data prospectively collected?

3. Are the main prognostic patient and tumor characteristics presented?

4. Is the method used for determination of protein expression specified?

Criteria for immunohistochemistry:

Is the immunohistochemical staining protocol specified? ${ }^{\mathrm{b}}$

Were stainings evaluated by $>1$ observer?

1

1

Criteria for RT-PCR:

Is the RNA isolation method and cDNA synthesis specified?

Is the PCR protocol specified?

1

1

5. Is the study endpoint defined?

6. Is the time of follow up specified?

7. Is loss during analysis or follow up described?

Total

${ }^{a}$ At least four of the following characteristics: age at diagnosis, tumor stage, tumor location, differentiation grade and residual tumor after primary surgery. ${ }^{\mathrm{b}} \mathrm{At}$ least four of the following criteria: antigen retrieval, primary antibody, dilution, detection method, cut-off value for positive expression: ${ }^{\mathrm{c} A t}$ least the primers used and the annealing temperature or number of cycles.

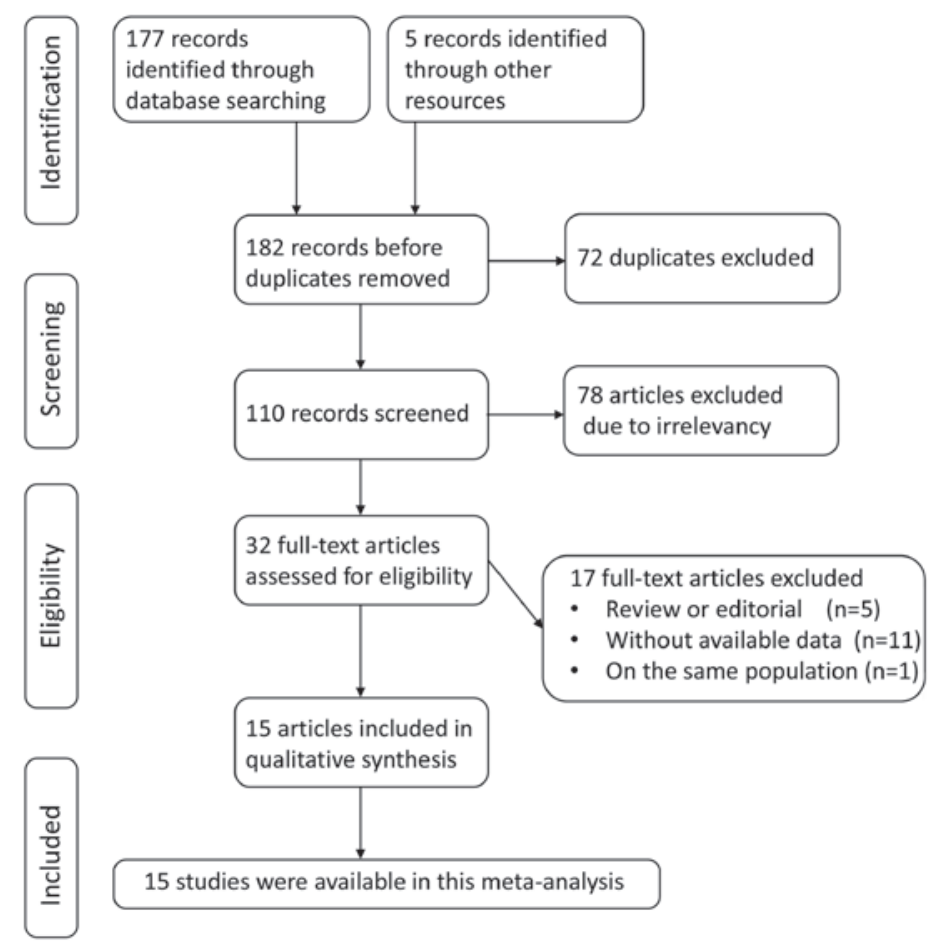

Figure 1. Flowchart of articles identified with criteria for inclusion and exclusion.

displayed in Fig. 5, the phenomenon that high ERCC1 expression was associated with a poor prognosis was first observed in the study reported by Squires et al (26) in 2013 (HR 1.48;
95\% CI 1.01-2.17). Following that, only one study was added cumulatively, resulting in an overall effect estimate of 1.48 (95\% CI 1.02-2.13). 


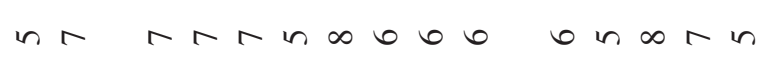

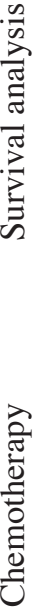

芯

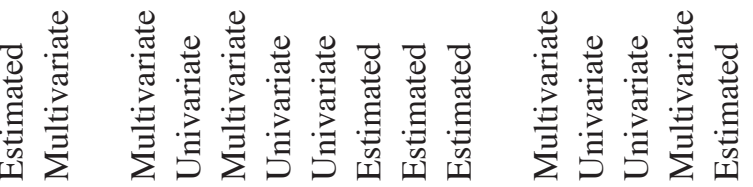

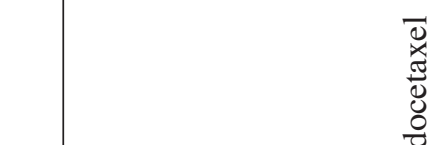

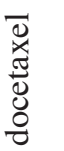

is

它

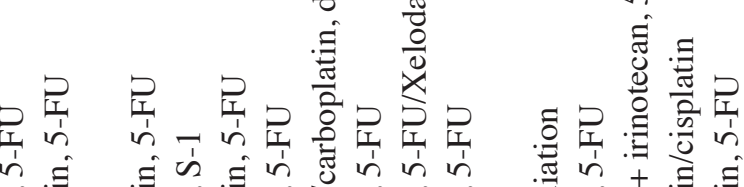

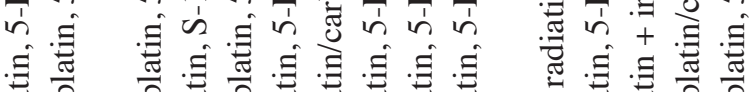

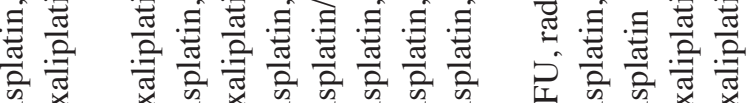

Uి

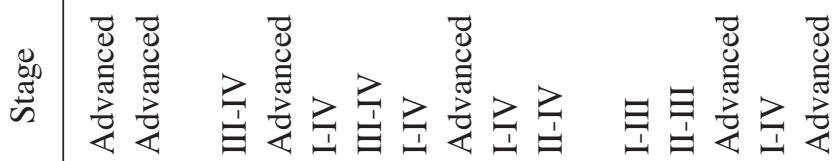

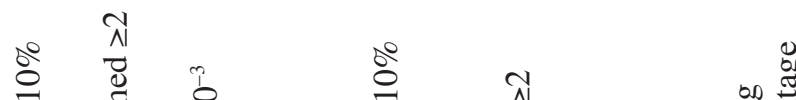

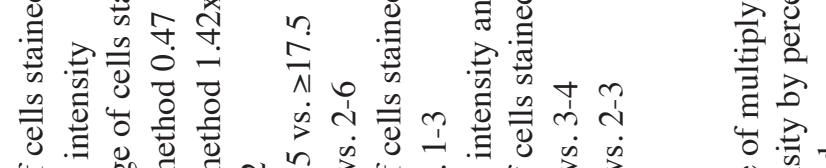

岂

4.00

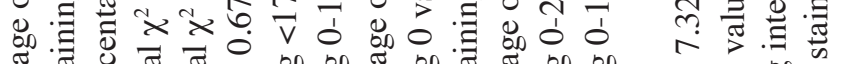

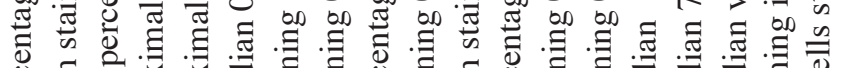

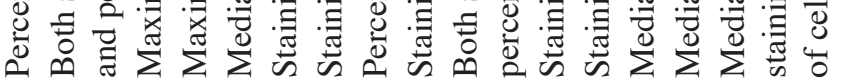

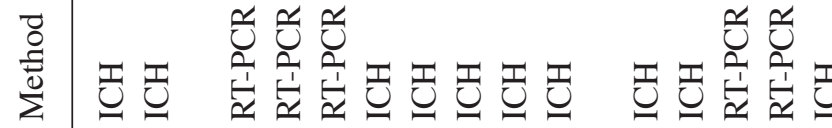

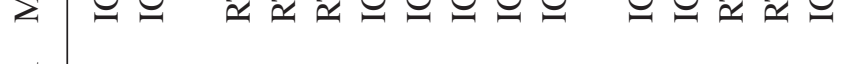

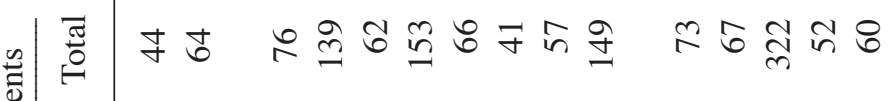

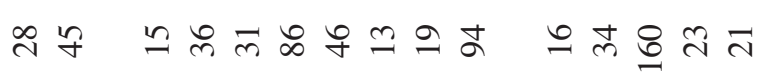

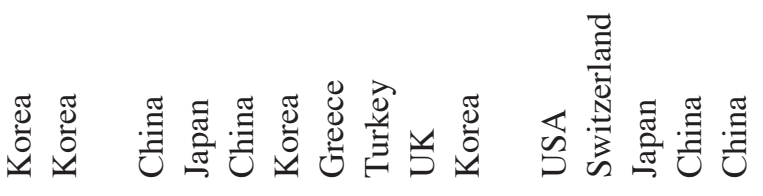

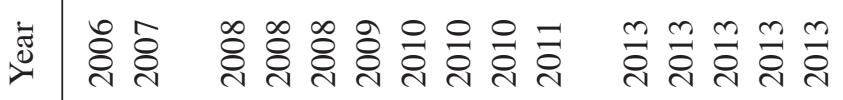

ลิ

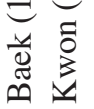

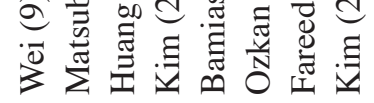

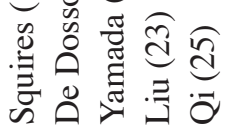


Table III. Stratified analysis of excision repair cross-complementation group 1 expression with overall survival.

\begin{tabular}{|c|c|c|c|c|c|c|c|}
\hline \multirow[b]{2}{*}{ Variables } & \multirow[b]{2}{*}{$\mathrm{n}^{\mathrm{a}}$} & \multicolumn{2}{|c|}{ Pooled HR (95\% CI) } & \multicolumn{3}{|c|}{ Heterogeneity test } & \multirow{2}{*}{$\begin{array}{c}\text { Meta-regression } \\
\text { P-value }\end{array}$} \\
\hline & & Fixed & Random & Q & P-value ${ }^{b}$ & $\mathrm{I}^{2}(\%)$ & \\
\hline Location & & & & & & & 0.759 \\
\hline Asian & 11 & $1.38(1.19,1.59)$ & $1.54(0.99,2.38)$ & 75.37 & $<0.001$ & 86.7 & \\
\hline Non-Asian & 4 & $1.28(0.88,1.86)$ & $1.31(0.63,2.75)$ & 10.81 & 0.013 & 72.3 & \\
\hline Method & & & & & & & 0.044 \\
\hline $\mathrm{IHC}$ & 10 & $0.99(0.82,1.21)$ & $1.09(0.69,1.72)$ & 44.58 & $<0.001$ & 79.8 & \\
\hline RT-RCR & 5 & $1.82(1.51,2.20)$ & $2.57(1.49,4.45)$ & 22.78 & $<0.001$ & 82.4 & \\
\hline Sample size & & & & & & & 0.262 \\
\hline$<100$ & 11 & $1.72(1.39,2.14)$ & $1.74(1.08,2.80)$ & 47.53 & $<0.001$ & 79.0 & \\
\hline$\geq 100$ & 4 & $1.16(0.97,1.39)$ & $1.02(0.55,1.91)$ & 31.14 & $<0.001$ & 90.4 & \\
\hline HR estimated & & & & & & & 0.304 \\
\hline Directly obtained & 10 & $1.50(1.28,1.76)$ & $1.73(1.13,2.64)$ & 52.25 & $<0.001$ & 82.8 & \\
\hline Indirectly obtained & 5 & $1.02(0.77,1.34)$ & $1.08(0.51,2.29)$ & 28.27 & $<0.001$ & 85.9 & \\
\hline Quality score & & & & & & & 0.177 \\
\hline $5-6$ & 8 & $0.98(0.78,1.22)$ & $1.12(0.66,1.89)$ & 36.49 & $<0.001$ & 80.8 & \\
\hline $7-8$ & 7 & $1.68(1.41,2.00)$ & $1.99(1.22,3.26)$ & 35.66 & $<0.001$ & 83.2 & \\
\hline
\end{tabular}

${ }^{\mathrm{a} N u m b e r}$ of comparisons. ${ }^{\mathrm{b}} \mathrm{P}$-value of Q-test for heterogeneity test. IHC, immunohistochemistry; RT-PCR, reverse transcription-polymerase chain reaction; HR, hazard ratio.

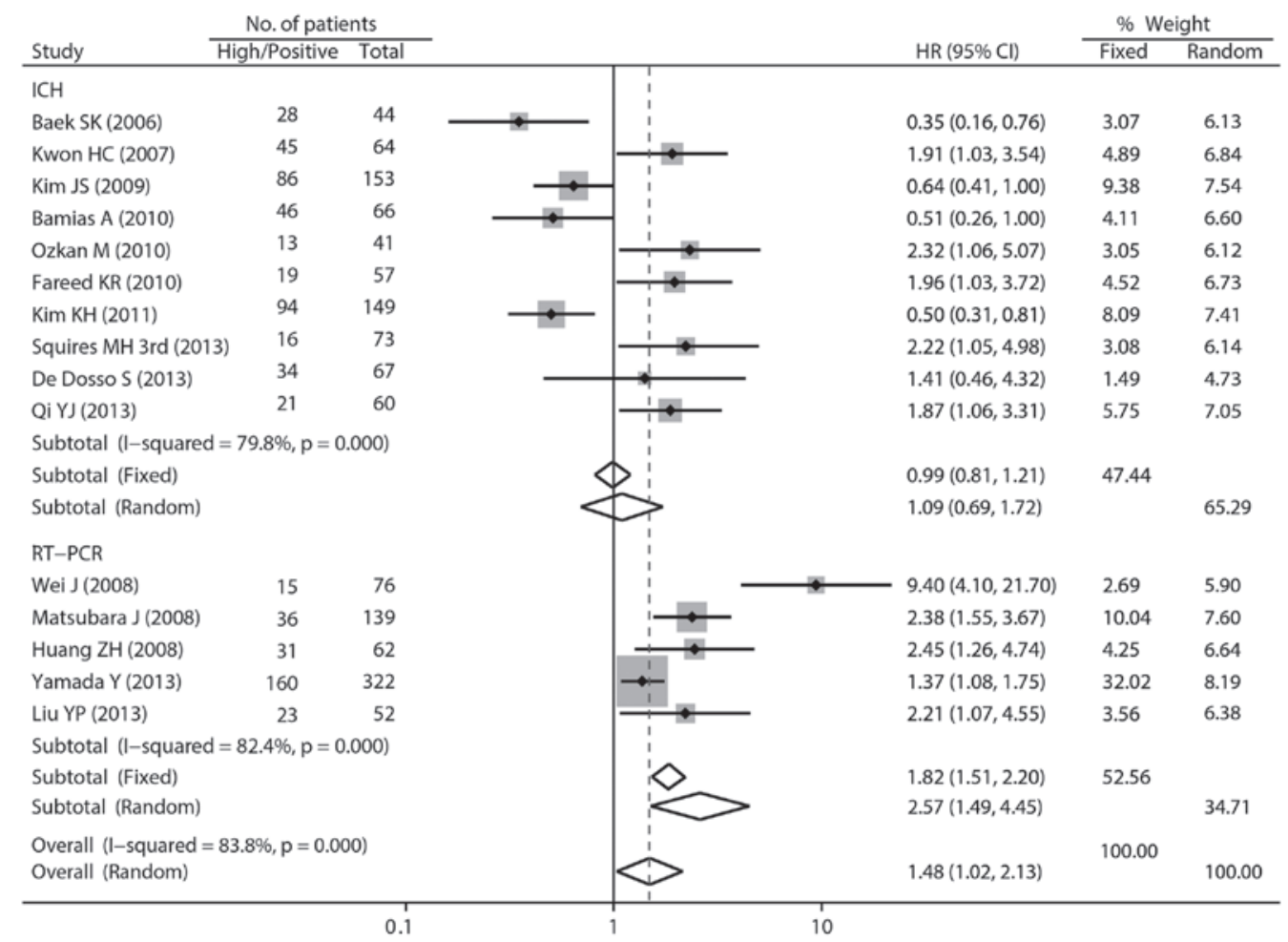

Figure 2. Pooled HRs for overall survival in patients with gastric cancer. The size of each square is proportional to the weight of the study (inverse of variance). HR, hazard ratio; CI, confidence interval; ICH, immunohistochemistry; RT-PCR, reverse transcription polymerase chain reaction.

Publication bias. The shape of the funnel plot did not exhibit any evident asymmetry (Fig. 6). The Begg's and Egger's tests indicated no evidence of publication bias $(\mathrm{P}=0.276$ for Begg's test; $\mathrm{P}=0.559$ for Egger's test).

\section{Discussion}

The identification of molecular biomarkers with prognostic value for GC is clinically useful. In this meta-analysis, the 


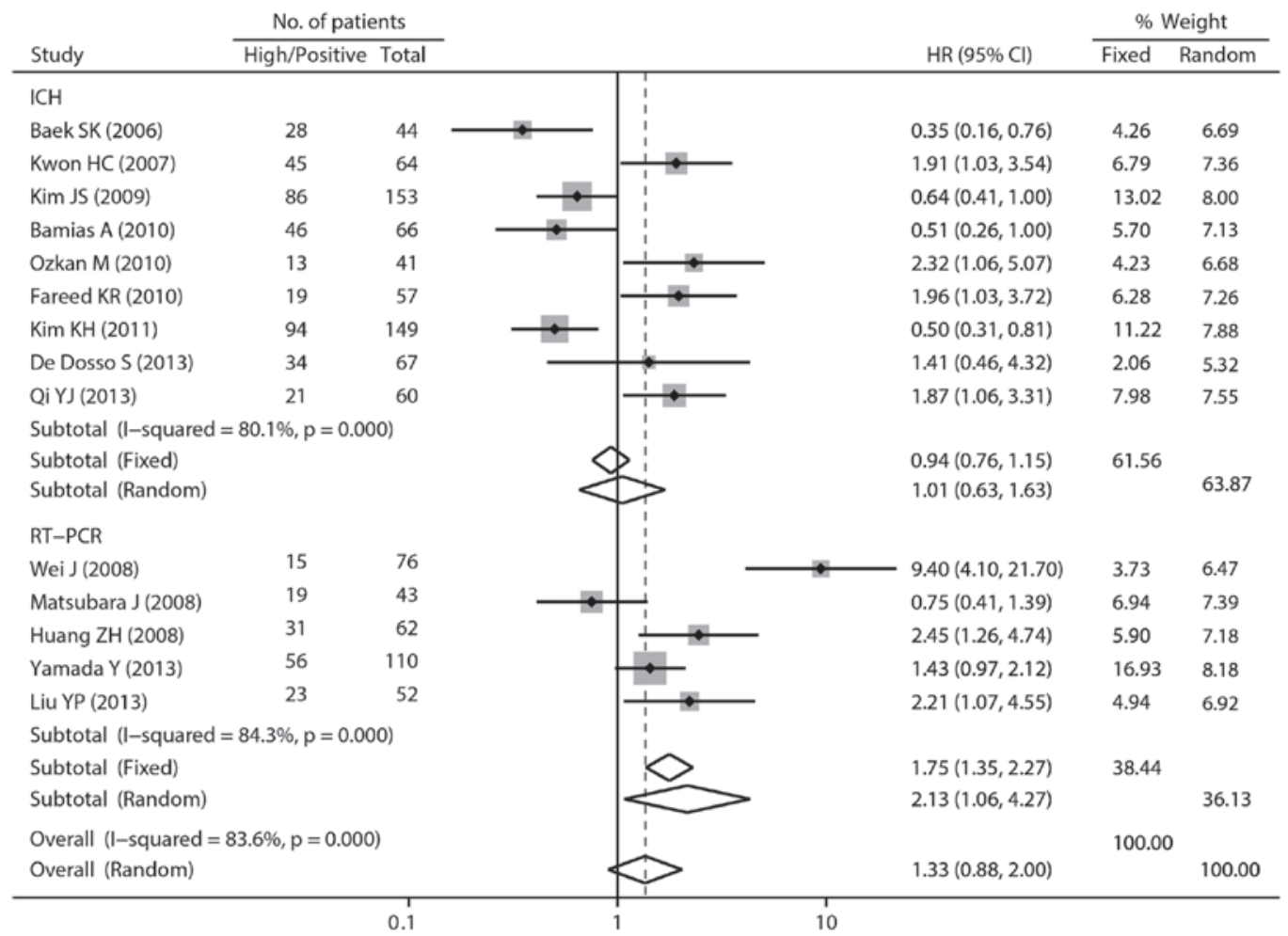

Figure 3. Pooled HRs for OS in GC patients receiving platinum-based chemotherapy. The size of each square is proportional to the weight of the study (inverse of variance). HR, hazard ratio; CI, confidence interval; OS, overall survival; GC, gastric cancer; ICH, immunohistochemistry; RT-PCR, reverse transcription polymerase chain reaction.

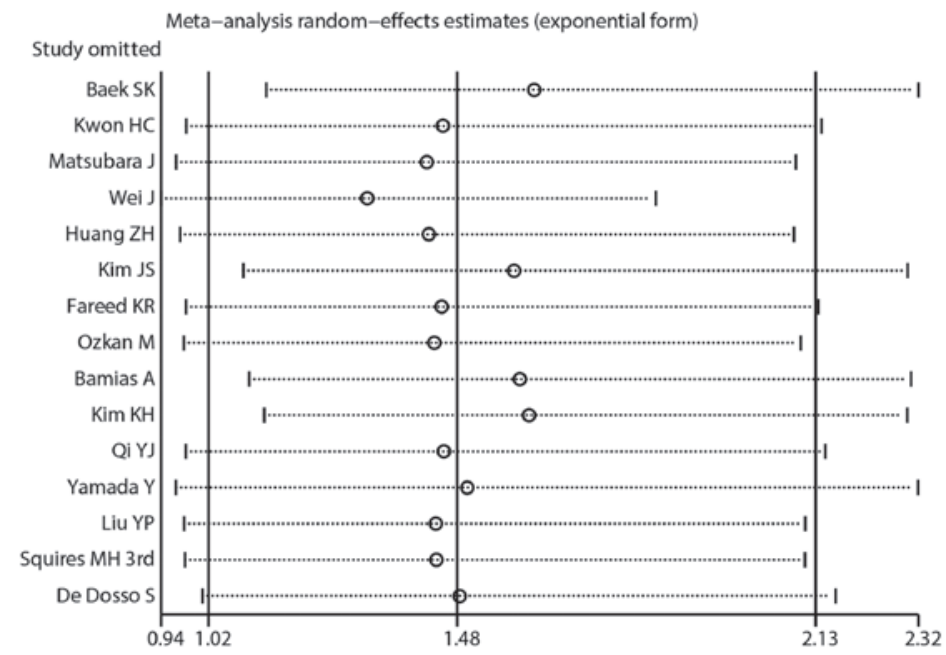

Figure 4. Influence analysis of the pooled hazard ratio for overall survival. Meta-analysis random effects estimates (exponential form) were used. Results were computed by omitting each study (on the left) in turn. The two ends of every broken line represented the $95 \%$ confidence interval.

effects of ERCC1 expression on the OS for GC were evaluated. The results indicate that high/positive ERCC1 expression is a significant poor prognostic factor for GC with chemotherapy regardless of the treatment regimen, compared with low/negative ERCC1 expression. Low mRNA levels of ERCC1 may be associated with a significant favorable OS benefit for platinum-based chemotherapy.

Numerous studies have reported that high/positive ERCC1 expression is associated with the prognosis of other types of cancer, including non-small cell lung (27), bladder (28), colorectal (29) and breast cancer (30). In addition, polymorphisms of ERCC1 have been found to affect OS in the platinum-based treatment of patients with GC (31). Overall, aberrant expression of ERCC1 appears to be associated with cancer risk. The biological role of ERCC1 may partly explain its poor prognosis. Cytotoxicity from platinum drugs leads to the formation of platinum DNA adducts, whereas ERCC1 acts to remove these bulky adducts and repair DNA double-strand damage. Furthermore, a high level of ERCC1 has been demonstrated to confer resistance to platinum agents and reconstitutes the ability of the cell to remove cisplatin from cellular DNA in an animal model (32). The aberrant methylation of DNA 


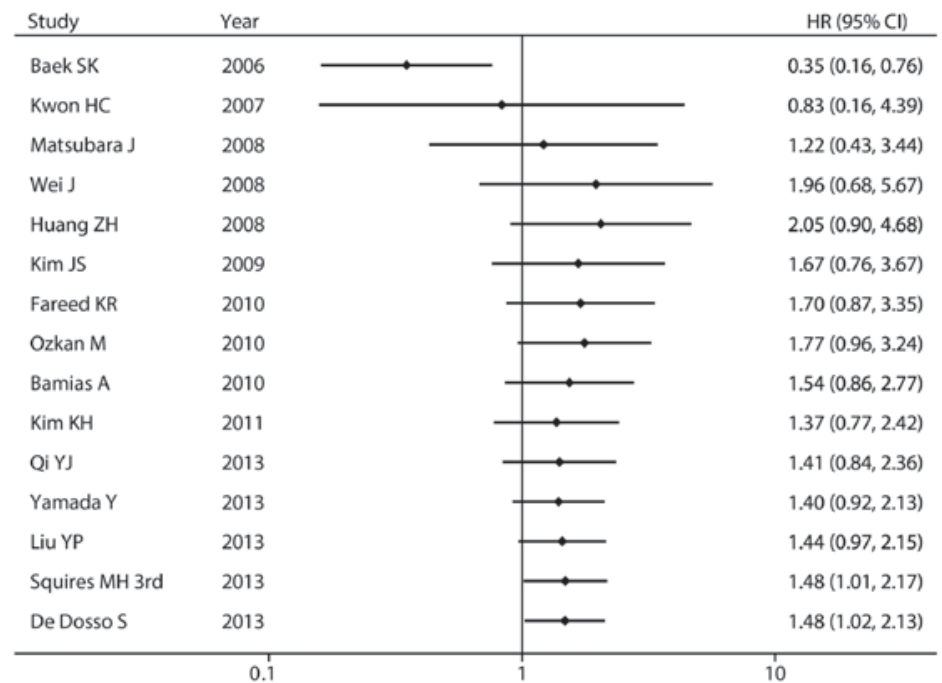

Figure 5. Cumulative meta-analysis showing the time-tendency of the HR for OS. HR, hazard ratio; CI, confidence interval, OS, overall survival.

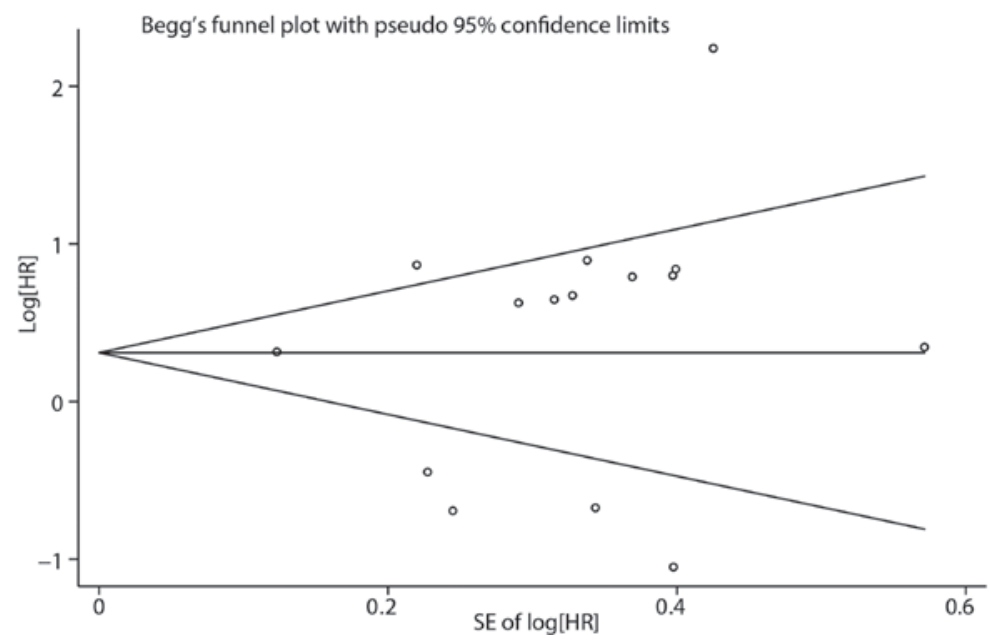

Figure 6. Funnel plot of the estimated publication bias of the included studies. HR, hazard ratio; SE, standard error.

repair genes including ERCC1 has also been reported to affect the sensitivity to chemotherapeutic agents $(33,34)$. The current results indicate that for the patients who received platinum-based chemotherapy, the risk of mortality increased with a high/positive expression of ERCC1 compared with the risk with low/negative ERCC1 expression.

To evaluate the effectiveness of different assessment methods, HRs were pooled from the IHC- or RT-PCR-based methods separately. In the present meta-analysis, RT-PCR appeared to be better than IHC in predicting OS for GC. ERCC1 expression using the IHC method was categorized by a visual grading system based on the staining intensity and percentage of cells stained, resulting in objectivity in certain circumstances. The ERCC1 mRNA levels were assessed with RT-PCR, which is a sensitive and quantitative method. This may one of the reasons why RT-PCR is more effective than IHC; however, the total sample size of the RT-PCR group was smaller than that of the IHC group (343 vs. 701). However, ERCC1 plays its role at the protein level. As is well-known, numerous factors can impact mRNA transcription. Notably, subgroup analyses demonstrated that the decreased survival associated with high ERCC1 expression was pronounced among high-quality-score studies. There is an urgent requirement for large-scale clinical studies to confirm these findings.

To the best of our knowledge, this was the first meta-analysis to evaluate the association between ERCC1 expression and the survival of patients with GC. There are, however, the following limitations to consider. Firstly, heterogeneity was significant in this meta-analysis. Although meta-regression analysis was used to clarify the source of heterogeneity, it was not successful. Additionally, sensitivity analysis did not help to find the source of heterogeneity. Secondly, where there were no directly obtained HRs in the studies, the estimated HRs were calculated from the data provided or extrapolated from the survival curves. The estimated HRs may be less reliable than those obtained directly from the papers. Thirdly, the cutoff values among these studies were different: Even in the IHC or RT-PCR subgroups the cutoff values were not unified. Studies with the same cutoff are, therefore, warranted to generate a more definitive conclusion. Fourthly, the cumulative meta-analysis presented significant associations until 2013, suggesting that this finding was not very robust with time. 
Finally, though no publication bias was detected in the present study, it could not be neglected. Since negative studies are often not published, and if these studies are published, they are often reported in a simplified way, this leads to difficulty in retrieving these data.

In conclusion, high/positive ERCC1 expression may be a poor prognostic factor for patients with GC. Due to the conferred resistance to platinum drugs, patients with high/positive ERCC1 expression (particularly with high ERCC1 mRNA levels) do not seem to benefit from platinum-based chemotherapy. Large scale and well-designed prospective studies are required to confirm the present findings.

\section{References}

1. Jemal A, Bray F, Center MM, Ferlay J, Ward E and Forman D: Global cancer statistics. CA Cancer J Clin 61: 69-90, 2011.

2. Mari E, Floriani I, Tinazzi A, et al: Efficacy of adjuvant chemotherapy after curative resection for gastric cancer: a meta-analysis of published randomised trials. A study of the GISCAD (Gruppo Italiano per lo Studio dei Carcinomi dell'Apparato Digerente). Ann Oncol 11: 837-843, 2000.

3. Martin LP, Hamilton TC and Schilder RJ: Platinum resistance: the role of DNA repair pathways. Clin Cancer Res 14: 1291-1295, 2008.

4. Sijbers AM, de Laat WL, Ariza RR, et al: Xeroderma pigmentosum group $\mathrm{F}$ caused by a defect in a structure-specific DNA repair endonuclease. Cell 86: 811-822, 1996.

5. Friboulet L, Olaussen KA, Pignon JP, et al: ERCC1 isoform expression and DNA repair in non-small-cell lung cancer. N Engl J Med 368: 1101-1110, 2013.

6. Chen WW, Wang F and Xu RH: Platinum-based versus non-platinum-based chemotherapy as first line treatment of inoperable, advanced gastric adenocarcinoma: a meta-analysis. PLoS One 8: e68974, 2013

7. Richards D, McCollum D, Wilfong L, et al: Phase II trial of docetaxel and oxaliplatin in patients with advanced gastric cancer and/or adenocarcinoma of the gastroesophageal junction. Ann Oncol 19: 104-108, 2008.

8. Yamada Y, Boku N, Nishina T, et al: Impact of excision repair cross-complementing gene 1 (ERCC1) on the outcomes of patients with advanced gastric cancer: correlative study in Japan Clinical Oncology Group Trial JCOG9912. Ann Oncol 24 2560-2565, 2013.

9. Wei J, Zou Z, Qian X, et al: ERCC1 mRNA levels and survival of advanced gastric cancer patients treated with a modified FOLFOX regimen. Br J Cancer 98: 1398-1402, 2008.

10. Matsubara J, Nishina T, Yamada Y, et al: Impacts of excision repair cross-complementing gene 1 (ERCC1), dihydropyrimidine dehydrogenase, and epidermal growth factor receptor on the outcomes of patients with advanced gastric cancer. Br J Cancer 98: 832-839, 2008.

11. Tierney JF, Stewart LA, Ghersi D, Burdett S and Sydes MR: Practical methods for incorporating summary time-to-event data into meta-analysis. Trials 8: 16, 2007.

12. de Graeff P, Crijns AP, de Jong S, et al: Modest effect of p53, EGFR and HER-2/neu on prognosis in epithelial ovarian cancer: a meta-analysis. Br J Cancer 101: 149-159, 2009.

13. Gooden MJ, de Bock GH, Leffers N, Daemen T and Nijman HW: The prognostic influence of tumour-infiltrating lymphocytes in cancer: a systematic review with meta-analysis. Br J Cancer 105: 93-103, 2011.

14. Egger M, Davey Smith G, Schneider M and Minder C: Bias in meta-analysis detected by a simple, graphical test. BMJ 315: 629-634, 1997.

15. Baek SK, Kim SY, Lee JJ, Kim YW, Yoon HJ and Cho KS: Increased ERCC expression correlates with improved outcome of patients treated with cisplatin as an adjuvant therapy for curatively resected gastric cancer. Cancer Res Treat 38: 19-24, 2006.
16. Bamias A, Karina M, Papakostas P, et al: A randomized phase III study of adjuvant platinum/docetaxel chemotherapy with or without radiation therapy in patients with gastric cancer. Cancer Chemother Pharmacol 65: 1009-1021, 2010.

17. De Dosso S, Zanellato E, Nucifora M, et al: ERCC1 predicts outcome in patients with gastric cancer treated with adjuvant cisplatin-based chemotherapy. Cancer Chemother Pharmacol 72: 159-165, 2013.

18. Fareed KR, Al-Attar A, Soomro IN, et al: Tumour regression and ERCC1 nuclear protein expression predict clinical outcome in patients with gastro-oesophageal cancer treated with neoadjuvant chemotherapy. Br J Cancer 102: 1600-1607, 2010.

19. Huang ZH, Hua D, Du X, et al: ERCC1 polymorphism, expression and clinical outcome of oxaliplatin-based adjuvant chemotherapy in gastric cancer. World J Gastroenterol 14: 6401-6407, 2008.

20. Kim JS, Kim MA, Kim TM, et al: Biomarker analysis in stage III-IV (M0) gastric cancer patients who received curative surgery followed by adjuvant 5-fluorouracil and cisplatin chemotherapy: epidermal growth factor receptor (EGFR) associated with favourable survival. Br J Cancer 100: 732-738, 2009.

21. Kim KH, Kwon HC, Oh SY, et al: Clinicopathologic significance of ERCC1, thymidylate synthase and glutathione S-transferase P1 expression for advanced gastric cancer patients receiving adjuvant 5-FU and cisplatin chemotherapy. Biomarkers 16: 74-82, 2011.

22. Kwon HC, Roh MS, Oh SY, et al: Prognostic value of expression of ERCC1, thymidylate synthase, and glutathione S-transferase P1 for 5-fluorouracil/oxaliplatin chemotherapy in advanced gastric cancer. Ann Oncol 18: 504-509, 2007.

23. Liu YP, Ling Y, Qi QF, et al: The effects of ERCC1 expression levels on the chemosensitivity of gastric cancer cells to platinum agents and survival in gastric cancer patients treated with oxaliplatin-based adjuvant chemotherapy. Oncol Lett 5: 935-942, 2013.

24. Ozkan M, Akbudak IH, Deniz K, et al: Prognostic value of excision repair cross-complementing gene 1 expression for cisplatin-based chemotherapy in advanced gastric cancer. Asian Pac J Cancer Prev 11: 181-185, 2010.

25. Qi YJ, Cui S, Yang YZ, et al: Excision repair cross-complementation group 1 codon 118 polymorphism, micro ribonucleic acid and protein expression, clinical outcome of the advanced gastric cancer response to first-line FOLFOX-4 in Qinghai-Tibetan plateau population. J Cancer Res Ther 9: 410-415, 2013.

26. Squires MH III, Fisher SB, Fisher KE, et al: Differential expression and prognostic value of ERCC1 and thymidylate synthase in resected gastric adenocarcinoma. Cancer 119: 3242-3250, 2013

27. Chen S, Zhang J, Wang R, Luo X and Chen H: The platinum-based treatments for advanced non-small cell lung cancer, is low/negative ERCC1 expression better than high/positive ERCC1 expression? A meta-analysis. Lung Cancer 70: 63-70, 2010.

28. Li S, Wu J, Chen Y, et al: ERCC1 expression levels predict the outcome of platinum-based chemotherapies in advanced bladder cancer: a meta-analysis. Anticancer Drugs 25: 106-114, 2014.

29. Huang MY, Tsai HL, Lin CH, et al: Predictive value of ERCC1, ERCC2, and XRCC1 overexpression for stage III colorectal cancer patients receiving FOLFOX-4 adjuvant chemotherapy. J Surg Oncol 108: 457-464, 2013.

30. Gerhard R, Carvalho A, Carneiro V, et al: Clinicopathological significance of ERCC1 expression in breast cancer. Pathol Res Pract 209: 331-336, 2013.

31. Yin M, Yan J, Martinez-Balibrea E, et al: ERCC1 and ERCC2 polymorphisms predict clinical outcomes of oxaliplatin-based chemotherapies in gastric and colorectal cancer: a systemic review and meta-analysis. Clin Cancer Res 17: 1632-1640, 2011.

32. Parker RJ, Eastman A, Bostick-Bruton F and Reed E: Acquired cisplatin resistance in human ovarian cancer cells is associated with enhanced repair of cisplatin-DNA lesions and reduced drug accumulation. J Clin Invest 87: 772-777, 1991.

33. Satoh A, Toyota M, Itoh F, et al: Epigenetic inactivation of CHFR and sensitivity to microtubule inhibitors in gastric cancer. Cancer Res 63: 8606-8613, 2003.

34. Chen HY, Shao CJ, Chen FR, Kwan AL and Chen ZP: Role of ERCC1 promoter hypermethylation in drug resistance to cisplatin in human gliomas. Int J Cancer 126: 1944-1954, 2010. 